\title{
Cetoacidose diabética e pancreatite secundárias à peg-asparaginase
}

Frederico Ribeiro Pires ${ }^{1,2}$ (D), Camila Abreu de Souza ${ }^{1}$ (D), Murilo Brito Luiz ${ }^{1}$ (D), Selma Harue Kawahara $^{1}$ (D), Luiz Claudio Gonçalves de Castro² (1)

\section{RESUMO}

Objetivos: Apresentar um caso raro de cetoacidose diabética (CAD) e pancreatite secundários ao uso de PEGasparaginase em paciente pediátrico em tratamento para leucemia linfoblástica aguda (LLA) e alertar quanto aos sinais que remetem a esses diagnósticos.

Descrição do caso: Adolescente do sexo feminino, 10 anos e 11 meses, em tratamento para LLA e uso prévio de PEG-asparaginase há seis dias da internação, admitida com choque hipotensivo grave e encaminhada à Unidade de Terapia Intensiva. Inicialmente o quadro foi interpretado como choque séptico. Em seguida a anamnese detalhada e os exames laboratoriais direcionaram para os diagnósticos de CAD e pancreatite, iniciando-se as intervenções específicas. Recebe alta hospitalar após 30 dias, sem necessidade de insulinoterapia, mas com reposição de enzimas pancreáticas.

Comentários: Geralmente, às crianças com LLA gravemente enfermos e leucopênicos, atribui-se apenas o diagnóstico de sepse, que é um diagnóstico prioritário. Entretanto, no grupo em uso de PEG-asparaginase, o pediatra emergencista deve estar alerta ao raciocínio diferencial envolvendo CAD e pancreatite, o que pode ser bem difícil inicialmente. $O$ alerta dos diagnósticos diferenciais do choque séptico, mesmo que raros, na assistência a pacientes oncológicos pediátricos, além da correta e pronta identificação do quadro e seu manejo apropriado, correlacionamse diretamente ao sucesso terapêutico e, em algumas situações, à sobrevivência do paciente.

Palavras-chave: PEG-asparaginase, Cetoacidose diabética, Pancreatite, Leucemia linfoblástica aguda.

1. Hospital da Criança de Brasília José Alencar. Unidade de Terapia Intensiva Pediátrica. Brasília, (DF), Brasil 2. Universidade de Brasília. Medicina da Criança e do Adolescente. Campus Universitário Darcy Ribeiro, UnB Area 1 - Asa Norte, Brasília, (DF), Brasill 


\section{INTRODUÇÃO}

A introdução da L-asparaginase ou de sua forma peguilada (PEG-asparaginase) nos protocolos de tratamento de indivíduos com leucemia linfoblástica aguda (LLA), contribuiu consideravelmente com o aumento das taxas de sucesso terapêutico e de melhora da sobrevida global desses pacientes, principalmente na faixa etária pediátrica ${ }^{1,2}$.

Atualmente, tem-se dado preferência à PEGasparaginase em detrimento da L-asparaginase, uma vez que a primeira possui maior meia-vida e menor imunogenicidade ${ }^{1-3}$. Entretanto, as duas formas dessa enzima apresentam potenciais efeitos adversos, como lesão hepática, eventos tromboembólicos venosos, hiperglicemia, hipertrigliceridemia e reação de hipersensibilidade ${ }^{3}$.

Em alguns indivíduos, o uso da L-asparaginase pode desencadear cetoacidose diabética (CAD), uma condição rara, com frequência estimada de $0,8 \%$ entre aqueles que usam esse quimioterápico ${ }^{4-9}$. Outro potencial efeito adverso é a pancreatite, descrita em 2 a $16 \%$ dos pacientes, cuja prevalência está correlacionada à dose cumulativa da droga. Essas duas condições podem coexistir, uma vez que a CAD pode decorrer da destruição citotóxica das ilhotas pancreáticas mediada pela pancreatite ${ }^{7}$. Geralmente essas situações adversas possuem curso limitado e os pacientes podem voltar a usar a medicação em momento futuro ${ }^{10-11}$.

No contexto da emergência pediátrica, o quadro agudo de dor abdominal e choque hipovolêmico em paciente oncológico neutropênico pode não ser tão claro para atribuí-lo à CAD e à pancreatite, o que pode levar o médico assistente a interpretar o quadro como choque séptico na maioria das vezes, que é o mais frequente nesse contexto, e intervir de acordo com esse diagnóstico. Um atraso no correto diagnóstico e na tomada de condutas adequadas conduz a um real aumento da morbimortalidade.

$O$ objetivo deste relato é apresentar um caso raro de CAD e pancreatite secundárias à $\mathrm{PEG}$-asparaginase em paciente pediátrico em tratamento para ШA e alertar quanto aos sinais e sintomas que podem remeter a esses diagnósticos. É uma condição pouco reportada, potencialmente fatal e geralmente não contemplada entre as hipóteses diagnósticas frente ao paciente em tratamento oncológico no cenário de emergência.

\section{DESCRIÇÃO DO CASO}

Sexo feminino, 10 anos e 11 meses de idade, massa de $41,6 \mathrm{~kg}$, branca, deu entrada em serviço de urgência e emergência pediátrica de um hospital terciário de Brasília-DF em mal estado geral, torporosa, com dor abdominal de forte intensidade, plenitude gástrica e episódios de fezes pastosas há dois dias da internação, associadas a desconforto respiratório em progressão, com padrão de Kussmaul à admissão. A paciente apresentava LLA do tipo $B$ e encontrava-se na fase 1 do Protocolo II BFM - Berlim-Frankfurt-Munique $2002^{12}$, adaptado para risco médio, sendo que havia realizado PEGasparaginase seis dias antes dessa intercorrência.

À admissão, a paciente encontrava-se em choque hipotensivo grave, classificado inicialmente como séptico. Foi iniciada expansão volêmica com cristaloide (total de $40 \mathrm{~mL} / \mathrm{kg}$, de forma rápida) e antibioticoterapia de amplo espectro com vancomicina e cefepima devido à internação recente e neutropenia febril.

Os exames laboratoriais de entrada encontramse na Tabela 1 e mostravam leucopenia grave, plaquetopenia, acidemia metabólica com ânions gap aumentados, normolactatemia e hiperglicemia.

A adolescente foi transferida à Unidade de Terapia Intensiva Pediátrica (UTIP), onde necessitou de nova expansão volêmica e titulação de adrenalina intravenosa até $0,4 \mathrm{mcg} / \mathrm{kg} / \mathrm{min}$, sem resposta adequada. Foi realizada dose de ataque de hidrocortisona $\left(100 \mathrm{mg} / \mathrm{m}^{2}\right)$ devido à refratariedade do choque às catecolaminas.

Na UTIP, evidenciou-se hálito cetônico e foi relatado pela genitora que a paciente mantinha polidipsia, polifagia e poliúria há três dias da admissão. Nesse período, havia sido observado também que a paciente apresentava perda ponderal. Esse contexto clínico e bioquímico da paciente permitiu o diagnóstico de CAD e foi iniciado tratamento conforme protocolo institucional. No dia seguinte, pela persistência da dor abdominal intensa e aumento de amilase sérica (ascensão de 108 para $778 \mathrm{U} / \mathrm{L}$; referência 28 - 100U/L), foi realizada Tomografia Computadorizada de abdome total, que descreveu aspectos compatíveis com pancreatite aguda (Figura 1).

Observa-se pâncreas discretamente aumentado (seta azul), com pequena área de hiporrealce em sua cauda compatível com pancreatite aguda e pequena área de necrose (seta vermelha). Presença de líquido peripancreático nos espaços pararrenais anteriores (seta verde).

Feito então o diagnóstico de pancreatite aguda segundo critérios de Suzuki et al. ${ }^{13}$, por 
Tabela 1. Exames laboratoriais da paciente.

\begin{tabular}{lcccc}
\hline Analito & Admissão & Dia 1 & Dia 10 & Referência \\
\hline Glicose & $391 \mathrm{mg} / \mathrm{dL}$ & $223 \mathrm{mg} / \mathrm{dL}$ & $62 \mathrm{mg} / \mathrm{dL}$ & Jejum: $70-99 \mathrm{mg} / \mathrm{dL}$ \\
Ureia & $27,2 \mathrm{mg} / \mathrm{dL}$ & $14 \mathrm{mg} / \mathrm{dL}$ & $21 \mathrm{mg} / \mathrm{dL}$ & $8-36 \mathrm{mg} / \mathrm{dL}$ \\
Creatinina & $0.47 \mathrm{mg} / \mathrm{dL}$ & $0,37 \mathrm{mg} / \mathrm{dL}$ & $0,06 \mathrm{mg} / \mathrm{dL}$ & $0,31-0,88 \mathrm{mg} / \mathrm{dL}$ \\
TGO (AST) & $21,5 \mathrm{UL}$ & $50 \mathrm{UL} / \mathrm{L}$ & $29 \mathrm{U} / \mathrm{L}$ & $5-36 \mathrm{U} / \mathrm{L}$ \\
TGP (ALT) & $52,7 \mathrm{U} / \mathrm{L}$ & $54 \mathrm{U} / \mathrm{L}$ & $57 \mathrm{U} / \mathrm{L}$ & $19-44 \mathrm{U} / \mathrm{L}$ \\
DHL & $331 \mathrm{U} / \mathrm{L}$ & $-103 \mathrm{U} / \mathrm{L}$ & $250-500 \mathrm{U} / \mathrm{L}$ \\
Lactato & $2 \mathrm{mmol} / \mathrm{L}$ & $4,9 \mathrm{mmol} / \mathrm{L}$ & $1,7 \mathrm{mmol} / \mathrm{L}$ & $0,63-2,44 \mathrm{mmol} / \mathrm{L}$ \\
Sódio & $131 \mathrm{mmol} / \mathrm{L}$ & $132 \mathrm{mmol} / \mathrm{L}$ & $140 \mathrm{mmol} / \mathrm{L}$ & $135-145 \mathrm{mmol} / \mathrm{L}$ \\
Potássio & $3,9 \mathrm{mmol} / \mathrm{L}$ & $2,2 \mathrm{mmol} / \mathrm{L}$ & $4,3 \mathrm{mmol} / \mathrm{L}$ & $3,3-4,6 \mathrm{mmol} / \mathrm{L}$ \\
Cloro & $108 \mathrm{mmol} / \mathrm{L}$ & $103 \mathrm{mmol} / \mathrm{L}$ & $101 \mathrm{mmol} / \mathrm{L}$ & $97-106 \mathrm{mmol} / \mathrm{L}$ \\
Cálcio iônico & $0,88 \mathrm{mmol} / \mathrm{L}$ & $0,99 \mathrm{mmol} / \mathrm{L}$ & $1,13 \mathrm{mmol} / \mathrm{L}$ & $1,13-1,32 \mathrm{mmol} / \mathrm{L}$ \\
Fósforo & $0,77 \mathrm{mmol} / \mathrm{L}$ & $0,49 \mathrm{mmol} / \mathrm{L}$ & $2,97 \mathrm{mmol} / \mathrm{L}$ & $3,3-5,3 \mathrm{mmol} / \mathrm{L}$ \\
Magnésio & $1,5 \mathrm{mg} / \mathrm{dL}$ & $0,93 \mathrm{mg} / \mathrm{dL}$ & $1,7 \mathrm{mg} / \mathrm{dL}$ & $1,6-2,6 \mathrm{mg} / \mathrm{dL}$ \\
pH & 7,00 & 7,27 & 7,48 & $7,35-7,5$ \\
Bicarbonato & $8,8 \mathrm{mEq} / \mathrm{L}$ & $13,4 \mathrm{mEq} / \mathrm{L}$ & $28 \mathrm{mEq} / \mathrm{L}$ & $22-29 \mathrm{mEq} / \mathrm{L}$ \\
Amilase & $108 \mathrm{U} / \mathrm{L}$ & $778 \mathrm{U} / \mathrm{L}$ & $49 \mathrm{U} / \mathrm{L}$ & $28-100 \mathrm{U} / \mathrm{L}$ \\
Albumina & $2,4 \mathrm{~g} / \mathrm{dL}$ & $2,0 \mathrm{~g} / \mathrm{dL}$ & $2,15 \mathrm{~g} / \mathrm{dL}$ & $2,9-4,7 \mathrm{~g} / \mathrm{dL}$ \\
HB/ Hematócrito & $13,8 \mathrm{~g} / \mathrm{dL} / 41 \%$ & $13,3 \mathrm{dL} / \mathrm{dL} / 39 \%$ & $9,7 \mathrm{~g} / \mathrm{dL} / 30,7 \%$ & $12-16 \mathrm{~g} / \mathrm{dL} / 36-46 \%$ \\
Leucócitos & 650 & 390 & 10.060 & $5.000-10.000 \mathrm{cel} / \mu \mathrm{L}$ \\
Neutrófilos & 150 & $S .260$ & $1.500-6.000 \mathrm{cel} / \mu \mathrm{L}$ \\
Plaquetas & $18.000 / \mu \mathrm{L}$ & $42.000 / \mu \mathrm{L}$ & $163.000 / \mu \mathrm{L}$ & $140.000-400.000 / \mu \mathrm{LL}$ \\
PCR & & $6,8 \mathrm{mg} / \mathrm{dL}$ & $1,75 \mathrm{mg} / \mathrm{dL}$ & $<, 5 \mathrm{mg} / \mathrm{dL}$ \\
\hline
\end{tabular}

AST: Aspartato aminotransferase; ALT: Alanina aminotransferase; DHL: Desidrogenase lática; HB: Hemoglobina; PCR: Proteína C Reativa.

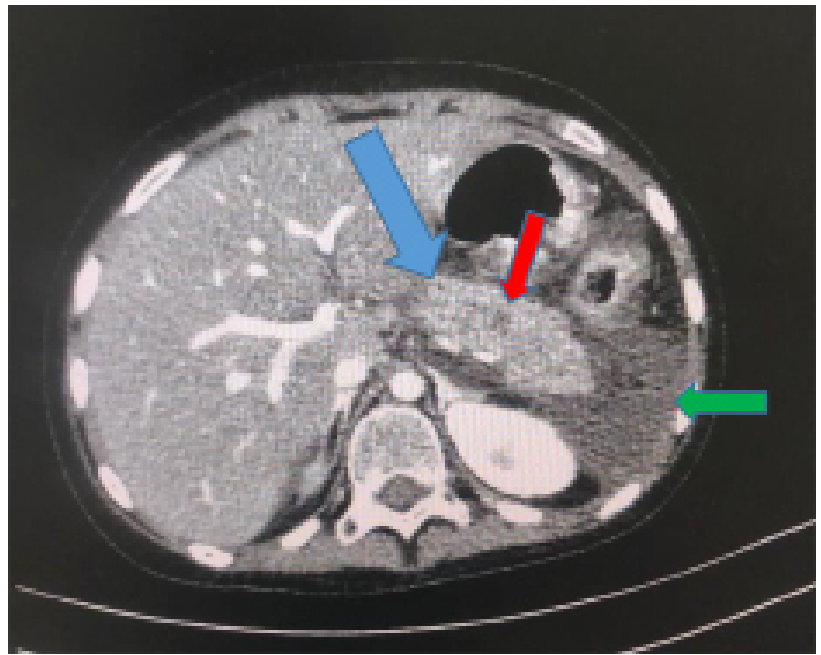

Figura 1. Tomografia Computadorizada (TC) de abdome da paciente após uso de contraste endovenoso.

ter a combinação de manifestações clínicas (dor abdominal intensa, choque hipovolêmico e vômitos), aumento expressivo de amilase e achado radiológico típico. Um aspecto importante quanto à pancreatite induzida por asparaginase é a ausência de hiperamilasemia na fase inicial em metade dos pacientes, como foi constatado no primeiro dia de internação. Esse fato é atribuído à deficiência da síntese proteica induzida pela asparaginase ${ }^{14}$.

Dentro desse contexto amplo, a paciente foi conduzida de acordo com os protocolos de tratamento da pancreatite aguda, CAD e choque hipovolêmico grave adotados pelo serviço. Apresentou adequada recuperação e recebeu alta da UTIP após dez dias, com bom controle glicêmico sem necessidade do uso de insulina, sem dor abdominal, com alimentação via sonda enteral e em recuperação medular pósquimioterapia. Após 30 dias de internação na enfermaria, recebeu alta com alimentação via oral estabelecida, mas ainda dependente de reposição de enzimas pancreáticas. Manteve-se sem necessidade de insulinoterapia. No fim da internação, foram obtidos os termos de consentimento e assentimento com a paciente e responsável, sendo posteriormente obtido o parecer consubstanciado do CEP (CAAE 33296420.8.0000.0025).

\section{DISCUSSÃO}

A LLA caracteriza-se como proliferação maciça de células linfoides imaturas na medula óssea e é o câncer mais comum da faixa etária pediátrica ${ }^{3}$. As taxas de cura atualmente registradas entre pacientes pediátricos com LLA são superiores a $90 \%$ em vários centros ${ }^{6}$, graças ao diagnóstico precoce e aos atuais protocolos terapêuticos, sendo a asparaginase um dos principais quimioterápicos responsáveis por essa resposta ${ }^{1-3}$. A asparaginase é uma enzima aminohidrolase que quebra a molécula de asparagina (através da catalisação da reação de transformação do aminoácido L-Asparagina em ácido aspártico) e esse processo é fundamental para desencadear apoptose das células leucêmicas. Isso acontece porque essas células, diferente das 
demais células do organismo, são incapazes de sintetizar a asparagina por não possuírem a asparagina sintetase ${ }^{3,15}$. Sem asparagina, as células leucêmicas não sobrevivem, uma vez que esse aminoácido é necessário à síntese proteica atrelada ao funcionamento e manutenção celular ${ }^{8-9}$.

As formulações de asparaginase disponíveis para o tratamento da LLA são a L-asparaginase derivada da Escherichia coli, a L-asparaginase derivada da Erwinia chrysanthemi e PEG-asparaginase (conjugado succinimidil monoetoxipolietileno glicol de Escherichia coli L-Asparaginase). Atualmente tem-se dado preferência ao uso da PEG-Asparaginase em relação à L-asparaginase, uma vez que as características farmacocinéticas da primeira permitem uma posologia com maior intervalo entre as doses pela sua maior meiavida $(5,7 \pm 3,2$ dias em comparação a $1,3 \pm 0,4$ dias, respectivamente, em administração intramuscular), além de sua menor imunogenicidade ${ }^{1-3,7,10}$. No entanto, ambas as formas dessa enzima possuem potenciais efeitos adversos como hepatotoxicidade, pancreatite, tromboembolismo venoso, hiperglicemia, hipertrigliceridemia e $\mathrm{CAD}^{6-7}$. A hiperglicemia e pancreatite são relativamente comuns $\mathrm{s}^{4,6,10-11,15}$ e podem estar presentes em até $25 \%$ e $16 \%$ dos pacientes pediátricos, respectivamente. No entanto, CAD é extremamente rara e referida em $0,8 \%$ das crianças e adolescentes em uso de L-asparaginase, geralmente associada ao uso concomitante de corticosteroides e em vigência de infecção ${ }^{4,9}$. As descrições de cetoacidose diabética e pancreatite concomitantes associadas ao uso de asparaginase em crianças e adolescentes com LLA são ainda mais raras ${ }^{6,16}$.

Entre os mecanismos fisiopatológicos sugeridos para explicar a CAD secundária ao uso da asparaginase estão as ações citotóxicas da droga desencadeando diminuição da secreção de insulina, resistência à insulina e/ou excesso de síntese de glucagon ${ }^{14,17}$. A CAD pode também ser secundária à pancreatite induzida pelo fármaco. Esses processos estão relacionados aos distúrbios da sinalização intracelular dos íons $\mathrm{Ca}^{2+}$ induzidos pela asparaginase nas células normais, causando diminuição das concentrações de ATP intracelular e necrose celular ${ }^{18}$.

Como as células beta-pancreáticas precisam de asparagina para a síntese de insulina ${ }^{9}$ e o uso crônico de corticosteroide pode desencadear resistência à insulina ${ }^{6,9}$, essa terapia apresenta potencial interferência no metabolismo insulínico que se torna ainda mais crítico em contexto de pancreatite.
Estudos sugerem que a pancreatite induzida pela asparaginase pode ocorrer em uma mediana de tempo em torno de três dias após o uso de L-asparaginase e de 11 dias após o uso de PEGasparaginase ${ }^{19}$. Segundo essa expressiva coorte de acompanhamento de pacientes pediátricos com LLA e pancreatite induzida por asparaginase $(n=465)$ ao longo de 20 anos, a presença de complicações metabólicas, como CAD e pancreatite, e desfechos letais apresentaram correlação significativa com a idade, com mediana de idade do grupo que desenvolveu complicações de 10,5 anos e do grupo sem complicação de $6,1 \operatorname{anos}^{19}$. A mortalidade secundária à pancreatite nessa coorte foi de $2 \%$. Este estudo também descreve que a manutenção de necessidade de insulinoterapia e dores abdominais recorrentes estavam associadas à presença de pseudocistos pancreáticos.

Apesar da hiperamilasemia ser uma caraterística bioquímica da pancreatite, deve-se ter cuidado ao interpretar o quadro clínico quando ela não está presente nesse grupo de pacientes, pois a asparaginase diminui a síntese dessa enzima pelas células acinares do pâncreas, ou seja, a ausência de hiperamilasemia não descarta o diagnóstico de pancreatite nas fases iniciais ${ }^{14}$.

No caso relatado, a paciente apresentou pancreatite e CAD secundárias à PEG-asparaginase, levando-a ao choque hipovolêmico grave, interpretado e tratado inicialmente como choque séptico, uma condição completamente plausível e um diagnóstico realmente prioritário no contexto. Entretanto, a refratariedade do choque às medidas iniciais para sepse levou à investigação complementar e subsequente que evidenciou persistência da acidemia grave, hiperglicemia importante e cetonúria, as quais, associadas ao quadro clínico descrito pela genitora de que a paciente apresentava poliúria, polidipsia e hálito cetônico, e à constatação de desidratação e glicosúria, direcionaram ao diagnóstico de CAD e a oportuna intervenção terapêutica.

A discussão dessa rara evolução clínica no prontoatendimento do paciente pediátrico oncológico é um importante alerta à possibilidade de sua ocorrência e de uma pronta investigação pelo médico emergencista. Todavia, para investigar essa condição, deve-se conhecê-la e inseri-la entre os possíveis diagnósticos diferenciais. Geralmente, esses pacientes admitidos em estado de choque ao 
serviço de saúde, por estarem imunossuprimidos e neutropênicos, tendem a ser tratados apenas pela hipótese de choque séptico, ao menos inicialmente. Tal conduta tem sua explicação pela prevalência desse diagnóstico e pela alta mortalidade decorrente da sepse naqueles indivíduos com neutropenia febril. Entretanto, é muito importante a avaliação de todo o contexto do paciente, incluindo os possíveis efeitos adversos de tratamentos farmacológicos em curso, com a elaboração de uma lista de hipóteses diagnósticas mais inclusiva, principalmente em pacientes oncológicos com LLA em uso de uma das formas de asparaginase. Essa abordagem inicial permite uma investigação diagnóstica mais detalhada e, consequentemente, maior possibilidade de confirmação precoce do correto diagnóstico e da conduta terapêutica mais adequada ao caso.

Nesse contexto apresentado pela paciente, é importante considerarmos a presença de sinais que se superpõem às manifestações clínicas e bioquímicas do choque séptico, da pancreatite e da CAD, como queda do estado geral, torpor, instabilidade hemodinâmica hipovolêmica e respiratória, acidose metabólica e hiperglicemia. Entretanto, há aspectos que quando presentes remontam à possibilidade de CAD, como história de perda ponderal, polidipsia, poliúria em vigência de desidratação, percepção de hálito cetônico, cetonemia e cetonúria importantes. A suspeição de pancreatite é provida pela intensa dor abdominal em quadrantes superiores e pela importante defesa abdominal.

Desta forma, no atendimento de emergência de pacientes em tratamento oncológico em uso de asparaginase, indica-se uma investigação laboratorial mais ampla para avaliação de lesões pancreáticas e suas consequências metabólicas, envolvendo glicemia, cetonemia, gasometria, amilasemia, lipasemia, calcemia, glicosúria e cetonúria. Essas podem ser complementadas com imagem abdominal (tomografia computadorizada), de acordo com a clínica e achados bioquímicos.

O caso relatado ilustra aspectos importantes de serem contemplados no pronto-atendimento da criança oncológica em choque, uma vez que a LLA é a neoplasia mais frequente em pediatria e que o uso de PEGasparaginase ou $\mathrm{L}$-asparaginase faz parte de protocolos utilizados. Nessas situações, a CAD e pancreatite precisam estar entre os diagnósticos diferenciais a serem trabalhados pelo médico assistente, assim como o conhecimento de aspectos específicos da manifestação e da evolução dessas condições.

Esse cuidado em exercer suspeição e investigação diagnósticas mais inclusivas é essencial à assistência médica expandida, qualificada e compromissada às crianças e adolescentes em tratamento oncológico que necessitam de avaliação em cenário de urgência e emergência.

\section{CONCLUSÃO}

O pediatra deve ter o cuidado adicional em pensar na hipótese diagnóstica de CAD em um paciente oncológico que fez uso de PEG-asparaginase, uma vez que o choque e desidratação, em um paciente pancitopênico, geralmente só leva ao pensamento de choque séptico. Os diagnósticos diferenciais para CAD e pancreatite, nesse contexto clínico, podem ser os detalhes para a condução de sucesso em um caso grave e difícil de um paciente oncológico pediátrico.

\section{REFERÊNCIAS}

1. Marini BL, Perissinotti AJ, Bixby DL, Brown J, Burke PW. Catalyzing improvements in ALL therapy with asparaginase. Blood Rev. 2017;31(5):328-38.

2. Boissel N, Sender LS. Best practices in adolescent and young adult patients with acute lymphoblastic leukemia: a focus on asparaginase. J Adolesc Young Adult Oncol. 2015;4(3):118-28.

3. Heo YA, Syed YY, Keam SJ. Pegaspargase: a review in acute lymphoblastic leukaemia. Drugs. 2019;79(7):767-77.

4. Ahmad MH, Shafiq I. Diabetic ketoacidosis following PEG-asparaginase therapy. Endocrinol Diabetes Metab Case Rep. 2018;2018:18-0064.

5. Raja RA, Schmiegelow K, Sorensen DN, Frandsen TL. Asparaginase-associated pancreatitis is not predicted by hypertriglyceridemia or pancreatic enzyme levels in children with acute lymphoblastic leukemia. Pediatr Blood Cancer. 2017;64(1):32-8.

6. Quintanilla-Flores DL, Flores-Caballero MÁ, RodríguezGutiérrez R, Tamez-Pérez HE, González-González JG. Acute pancreatitis and diabetic ketoacidosis following L-asparaginase/prednisone therapy in acute lymphoblastic leukemia. Case Rep Oncol Med. 2014;2014:139169.

7. Galindo RJ, Yoon J, Devoe C, Myers AK. PEGasparaginase induced severe hypertriglyceridemia. Arch Endocrinol Metab. 2016;60(2):173-7.

8. Buie LW, Moore J, van Deventer H. Successful use of octreotide as a chemoprotectant for prevention of PEG- 
asparaginase-induced pancreatitis. Pharmacotherapy. 2014;34(8):e149-51.

9. Gifford G, Milliken S, Greenfield J. Diabetic ketoacidosis secondary to L-asparaginase in acute lymphoblastic leukaemia. Intern Med J. 2013;43(8):946-948.

10. Oparaji JA, Rose F, Okafor D, Howard A, Turner RL, Orabi AI et al. Risk Factors for Asparaginase-associated Pancreatitis: a systematic review. J Clin Gastroenterol. 2017;51(10):907-13.

11. Roberson JR, Raju S, Shelso J, Pui CH, Howard SC. Diabetic ketoacidosis during therapy for pediatric acute lymphoblastic leukemia. Pediatr Blood Cancer. 2008;50(6):1207-12.

12. Stary J, Zimmermann M, Campbell M, Castillo L, Dibar E, Donska S, et al. Intensive Chemotherapy for Childhood Acute Lymphoblastic Leukemia: Results of the Randomized Intercontinental Trial ALL IC-BFM 2002. Journal of Clinical Oncology 2014 32:3, 174-184.

13. Suzuki M, Sai J K, Shimizu T. Acute pancreatitis in children and adolescents. World J Gastrointest Pathophysiol. 2014;5(4):416-26. Review.

14. Minowa K, Suzuki M, Fujimura J, Saito M, Koh K, Kikuchi $A$, et al. L-asparaginase-induced pancreatic injury is associated with an imbalance in plasma amino acid levels. Drugs R D. 2012;12(2):49-55.

15. Raja RA, Schmiegelow $K$, Albertsen BK, Prunsild $K$, Zeller B, Vaitkeviciene G, Abrahamsson J, Heyman M,
Taskinen M, Harila-Saari A, Kanerva J, Frandsen TL; Nordic Society of Paediatric Haematology and Oncology (NOPHO) group. Asparaginase-associated pancreatitis in children with acute lymphoblastic leukaemia in the NOPHO ALL2008 protocol. Br J Haematol. 2014;165(1):126-33.

16. Jameel PZ, Lohiya S, Dongre A, Damke S, Lakhkar BB. Concurrent diabetic ketoacidosis and pancreatitis in Paediatric acute lymphoblastic leukemia receiving L-asparaginase. BMC Pediatr. 2020;20(1):228.

17. Tosur M, Viau-Colindres J, Astudillo M, Redondo MJ, Lyons SK. Medication-induced hyperglycemia: pediatric perspective. BMJ Open Diabetes Res Care. 2020;8(1):e000801.

18. Peng $\mathrm{S}$, Gerasimenko JV, Tsugorka T, Gryshchenko $\mathrm{O}$, Samarasinghe $\mathrm{S}$, Petersen $\mathrm{OH}$ et al. Calcium and adenosine triphosphate control of cellular pathology: asparaginase-induced pancreatitis elicited via proteaseactivated receptor 2 . Philos Trans R Soc Lond B Biol Sci. 2016;371(1700):20150423.

19. Wolthers BO, Frandsen $T L$, Baruchel A, Attarbaschi A, Barzilai S, Colombini A, Escherich G, Grell K, Inaba $H$, Kovacs G, Liang DC, Mateos M, Mondelaers V, Möricke A, Ociepa T, Samarasinghe $S$, Silverman LB, van der Sluis IM, Stanulla M, Vrooman LM, Yano M, Zapotocka E, Schmiegelow K; Ponte di Legno Toxicity Working Group. Asparaginase-associated pancreatitis in childhood acute lymphoblastic leukaemia: an observational Ponte di Legno Toxicity Working Group study. Lancet Oncol. 2017;18(9):1238-48. 


\section{Contribuição de cada autor}

1- Contribuição substancial no esboço do estudo ou na interpretação dos dados:

FRP, CAS, MBL, SHK, LCGC.

2- Participação na redação da versão preliminar: FRP, CAS, MBL, SHK, LCGC.

3- Participação na revisão e aprovação da versão final: FRP, CAS, MBL, SHK, LCGC.

4- Conformidade em ser responsável pela exatidão ou integridade de qualquer parte do estudo: FRP, CAS, MBL, SHK, LCGC.

\section{Declaração de conflito de interesse}

Os autores declaram não haver conflitos de interesse.

\section{Fonte financiadora do projeto}

Este trabalho não recebeu apoio financeiro.

\section{Justificativa da importância do trabalho}

Este trabalho demonstra a raridade e a importância do diagnóstico de cetoacidose diabética e pancreatite nos pacientes oncológicos que fizeram uso de PEG-asparaginase. A maioria deles chegam ao serviço de saúde em choque e neutropênicos, sendo aventada hipótese de choque séptico apenas. No entanto, devemos pensar sempre nos diferenciais acima para o melhor manejo desses pacientes já imunossuprimidos.

\section{Autor Correspondente:}

Frederico Ribeiro Pires

frederico_ripires@hotmail.com

Editor:

Prof. Dr. Paulo Henrique Manso

Recebido: $30 / 12 / 2020$

Aprovado: 28/04/2021 